\title{
Molecular Characterization of Pear 1-Aminocyclopropane-1-Carboxylate Synthase Gene Preferentially Expressed in Leaves
}

\author{
Haiyan Shi, Yuxing Zhang (Corresponding author) \& Wen Sun \\ College of Horticulture, Agricultural University of Hebei, Baoding 071001, China \\ E-mail: shyrainbow1980@yahoo.com.cn; jonsonzhyx@yahoo.com.cn \\ Liang Chen \\ Wuhan Botanical Garden, Chinese Academy of Sciences, Wuhan 430074, China \\ $\&$ \\ Key Laboratory of Plant Germplasm Enhancement and Specialty Agriculture \\ Chinese Academy of Sciences, Wuhan 430074, China \\ Yanan Su \& Diansheng Zhang \\ College of Horticulture, Agricultural University of Hebei, Baoding 071001, China
}

Received: December 31, 2011 Accepted: January 16, 2011 Online Published: April 17, 2012

doi:10.5539/jas.v4n6p72

URL: http://dx.doi.org/10.5539/jas.v4n6p72

This work is supported by The Education Department of Hebei Province Youth Fund (2010138)

\begin{abstract}
1-Aminocyclopropane-1-carboxylate (ACC) synthase catalyzes the conversion of S-adenosyl-L-methionine to $\mathrm{ACC}$ in the ethylene biosynthetic pathway. Lots of important ACC synthase genes have been isolated and characterized from the plant kingdom. In this study, a cDNA clone encoding putative ACC synthase was isolated from a cDNA library produced using mRNA from pear (Pyrus pyrifolia). The cDNA clone, designated PpACS1 (GenBank accession No. JQ284383), comprised an open reading frame of 1, 341 bp encoding a protein of 446 amino acids that shares high similarity with the known plant ACSs. Using PCR amplification techniques, a genomic clone corresponding to PpACS1 was isolated and shown to contain two introns with typical GT/AG boundaries defining the splice junctions. The PpACS1 gene product shared $97 \%$ identity with an ACC synthase from pear (Pyrus communis), and phylogenetic analyses clearly placed the gene product in the ACC synthase cluster of the plant ACS superfamily tree. RT-PCR analysis indicated that the PpACS1 gene was preferentially expressed in pear leaves. The transcript of PpACS1 gene was accumulated at relatively high levels in anthers. The expression signal was detected in shoot at relatively low levels, but none signal was detected in developing fruit of pear. These results suggested that the PpACS1 may participate in the regulation of ethylene production in pear leaves and anthers.
\end{abstract}

Keywords: Pear (Pyrus pyrifolia), Ethylene, 1-Aminocyclopropane-1-carboxylate (ACC) synthase, Gene expression

\section{Introduction}

Ethylene is a plant hormone regulating a wide range of physiological processes such as germination, growth, development, and senescence (Klee and Tieman, 2002). As a senescing hormone, it promotes leaf-yellowing, flower and leaf abscission, climacteric fruit ripening (Yang and Hoffman, 1984). The production of ethylene in higher plants is from S-adenosyl-L-methionine (SAM) via the intermediate 1-aminocyclopropane-1-carboxylic acid (ACC). The conversion of SAM to ACC is catalysed by ACC synthase, and the subsequent oxidation of ACC to ethylene is catalysed by ACC oxidase (Yang and Hoffman, 1984; Ververidis and John, 1991). 
ACC synthase (ACS), the rate-limiting enzyme of the ethylene biosynthesis pathway, is encoded by a multigene family. For example, there are eight $A C S$ genes in tomato, six in mung bean, five in potato, five from rice, and ten from Arabidopsis (Wang et al., 2005). Lots of important ACC synthase genes have been isolated and characterized from the plant kingdom. For examples, ACS genes were identified in pear (Pyrus communis) (El-Sharkawy et al., 2004), Japanese pear (Pyrus pyrifolia) (Itai et al., 1999), apple (Rosenfield et al., 1996; Wang et al., 2009), peach (Mathooko et al., 2001; Tatsuki et al., 2006), pineapple (Cazzonelli et al., 1998; Botella et al., 2000; Trusov et al., 2006), plum (El-Sharkawy et al., 2008), cucumber (Mathooko et al., 1999), watermelon (Salman-Minkov et al., 2008), Arabidopsis (Rodrigues-Pousada et al., 1993; Mayer et al., 1999), tomato (Nakatsuka et al., 1997), etc.

In watermelon, both CitACS1 and CitACS3 are expressed in floral tissue. CitACS1 is also expressed in vegetative tissue and it may be involved in cell growth processes. The CitACS3 gene is expressed in open flowers and in young staminate floral buds (male or hermaphrodite), but not in female flowers. CitACS3 is also up-regulated by ACC, and is likely to be involved in ethylene-regulated anther development. The expression of CitACS2 was not detected in vegetative or reproductive organs (Salman-Minkov et al., 2008). Silencing of ACACS2 causes delayed flowering in pineapple (Trusov et al., 2006). The expression of AtACS1 is under developmental control both in shoot and root. High expression was observed in young tissues and was switched off in mature tissues (Rodrigues-Pousada et al., 1993). However, little is known so far about the expression of pear ACS genes during early development. Here, isolation and characterization of one pear ACS gene and its expression pattern during early and fruit development are reported.

\section{Materials and Methods}

\subsection{Collection of Plant Materials}

Pear (Pyrus pyrifolia Nakai cv.Whangkeumbae) fruit was harvested at 120, 150, and 180 day-old after full bloom from a local orchard. The other tissues (such as shoots, leaves, petals, and anthers) were derived from the same pear trees from the local orchard. These samples were frozen immediately in liquid nitrogen, and then stored at $-80^{\circ} \mathrm{C}$ for RNA isolation.

\subsection{Construction of Pear cDNA Library and Isolation of PpACS1 cDNA}

Total RNA was extracted from pear tissues including shoots, leaves, petals, anthers, and fruit. Poly $(\mathrm{A})^{+} \mathrm{mRNA}$ was prepared from a pool of total RNA by using an mRNA purification kit (Qiagen). Complementary DNA was synthesized and cloned into the EcoR I-Xho I sites of the ZAP express vector and packaged using a ZAPcDNA Gigapack Gold III cloning kit (Stratagene) according to the manufacturer's instruction.

More than 3,000 cDNA clones were randomly selected from the pear cDNA library for sequencing. One PpACS clone with complete sequences was identified. The corresponding $P p A C S$ gene was amplified from the genomic DNA of pear by PCR, using Pfu DNA polymerase and gene-specific primers that were designed according to the sequence of PpACS cDNA. In total, one PpACS gene was obtained.

\subsection{RNA Isolation and RT-PCR Analysis}

Total RNA was isolated from shoots, leaves, petals, anthers, and developing fruit (120, 150, and 180 day-old after full bloom) of pear by the method described previously (Shi et al., 2009). A 2-4 g aliquot of each pear tissue was randomly collected from 3-10 plants for RNA isolation. The concentration and purity of total RNA were identified by NanoDrop spectrophotometry and agarose gel electrophoresis. RNA samples were stored at $80^{\circ} \mathrm{C}$ until use.

Expression profiling of the PpACS1 gene in different pear tissues (such as shoots, leaves, petals, and anthers) and during different stages of fruit development (including 120,150, and 180 day-old after full bloom fruit) was carried out by semi-quantitative RT-PCR. A pear polyubiquitin gene (PpUBI, GenBank accession no. AF195224) was used as a standard pear in the RT-PCRs. A two-step RT-PCR procedure was performed in the experiments using a previously described method (Shi et al., 2009). First, $2 \mu \mathrm{g}$ of purified total RNA using Qiagen RNeasy Mini kit was reversely transcribed into cDNAs by M-MLV reverse transcriptase according to the manufacturer's instructions. The cDNAs were used as templates for RT-PCR reactions using gene-specific primers (PpACS1 P1: 5'- CAAAGATCTTGGCCTTCCAGGCTT-3'; P2: 5'- CAGAAAGAGTAAATTGGGTTTTGTCC-3').

\subsection{DNA Sequencing and Protein Analysis}

The open reading frame (ORF) of PpACS1 gene and its deduced protein sequence were analyzed using DNASTAR software (DNAStar Co, Madison, WI, USA). The conserved domain was determined by NCBI Conserved Domain Search (http: //blast.ncbi.nlm.nih.gov/Blast.cgi). Sequence alignment of PpACS1 with other 
plant ACSs were performed using ClustalW (http: //www.ebi.ac.uk/clustalw/) and protein motif analysis using motif scan (http: //myhits.isb-sib.ch/cgi-bin/motif_scan). N-glycosylation of the putative pear ACS protein was investigated using NETNGLYC (http: //www.cbs. dtu.dk/services/NetNGlyc/). The evolutionary relationships of the ACS proteins were determined by MEGA3.1 software, based on minimum evolution from 1000 bootstrap replicates.

\subsection{Homology Modeling}

The 3D models of the whole and domains of the deduced PpACS1 protein have been built using the 3D structure IIAX chain 'A' as template (Lambert et al., 2002) and SWISS-MODEL Workspace. Figures were generated using rw32b2a (http://www.37c.com.cn/topic/004/netguide/zip/RasMol26.zip).

\section{Results}

\subsection{Isolation and Characterization of PpACS1 cDNA}

To isolate the genes that might be involved in the regulation of ethylene production, 3, 000 cDNA clones from the pear (Pyrus pyrifolia) cDNA library were randomly sequenced. One $A C S$ cDNA clone containing full length $A C S$ cDNA sequence was identified from these clones, designated as PpACS1 (GenBank accession no: JQ284383), encoding polypeptides consisting of 446 amino acids (50.00 kD, pI 5.55). The putative PpACS1 protein include 44 strongly basic(+) amino acids (K,R), 56 strongly acidic(-) amino acids (D,E), 147 hydrophobic amino acids (A,I,L,F,W,V), and 127 polar amino acids (N,C,Q,S,T,Y). Sequence alignment revealed that all of the predicted proteins encoded by these genes contain highly conserved Aminotransferase class I and II superfamily domain (Fig.1).

\subsection{Structural Analysis of the PpACS1 Protein}

Analysis of protein structure revealed that the predicted PpACS1 polypeptide contain the typical Aminotransferase class I and II domain and other conserved motifs (i.e. Aminotransferases class-I pyridoxal-phosphate attachment site, N-glycosylation site, cAMP- and cGMP-dependent protein kinase phosphorylation site). As shown in Fig.1, the typical Aminotransferase class I and II domains $\left(\mathrm{V}_{51}-\mathrm{I}_{435}\right)$ and Aminotransferases class-I pyridoxal-phosphate attachment sites $\left(\mathrm{S}_{280}-\mathrm{G}_{293}\right)$ are highly conserved among the plant ACSs. Interestingly, three substitutions at amino acid level occurred in PpACS1 compared with other pear ACS proteins (Fig.1). At position 74, the polar Ser was substituted by a polar Cys. At position 430, the hydrophobic Ile was substituted by a hydrophobic Val. The strongly basic (+) Lys at residues 338 was substituted by a polar Asn, suggesting that PpACS1 may have a different structure and function compared with other pear ACS variants. Through PpACS1 protein, there are only one $\mathrm{N}$-glycosylation site (PGSs; $\mathrm{N}$ in NxS/T motifs, positions $422)$ and cAMP- and cGMP-dependent protein kinase phosphorylation site $\left(\mathrm{R}_{307} \mathrm{RMS}_{310}\right)$. Sequence comparison revealed that PpACS1 shares the highest similarity (97\% identity) with PcACS2b (El-Sharkawy et al., 2004) and pPPACS2 (Itai et al., 1999). It shares relatively high homology ( $96 \%$ identity) with MdACS3a-1 and MdACS3a-2, but relatively low homology (83-95\% identities) with pPPACS1 (Itai et al., 1999) and other known plant ACSs (Fig.1). This conserved structure may be related to the maintenance of ACS functions for plant development.

To understand the protein structure in detail, we predicted the 3D structure of PpACS1 from ESyPred3D (Lambert et al., 2002). 3D homology model of PpACS1 has been built by using the 3D structure 1IAX chain 'A' as a template. We also used it to build the 3D structure of Aminotransferase class I and II domain. The whole modeling (Fig.2A/B) is restricted to residues $1-446$ of PpACS1, and shares $54.0 \%$ identity with the template sequence (using the ALIGN program). 3D model of Aminotransferase class I and II domain $\left(\mathrm{V}_{51}-\mathrm{I}_{435}\right.$ ) (Fig.2C/D) has been built using the $3 \mathrm{D}$ structure $1 \mathrm{IAX}$ chain ' $\mathrm{A}$ ' as the template. It shares $50.9 \%$ identity with the template sequence.

\subsection{Phylogenetic Relationship of PpACS Protein}

To investigate the evolutionary relationships of the pear ACS proteins with apple and Arabidopsis ACS proteins, all of the known pear ACSs, apple ACSs, and Arabidopsis ACSs were selected from GenBank for phylogenetic analysis. The ACS proteins can be divided into two groups. As shown in Fig.3, these proteins obviously split into two subgroups. PpACS1, PcACS2b (El-Sharkawy et al., 2004), PcACS2a (El-Sharkawy et al., 2004), pPPACS2 (Itai et al., 1999), MdACS3a-2, MdACS3a-1, MdACS3c (Wang et al., 2009), PcACS3a, MdACS3b (Wang et al., 2009) and AtACS7 (Mayer et al., 1999) form one subgroup, while PbACS1B, pPPACS1 (Itai et al., 1999) and PcACS1b (El-Sharkawy et al., 2004) together with the Arabidopsis ACS8 are located in the second branch of the tree. These results suggest that the divergence could have occurred before the differentiation of the plants. PpACS1 is located on the first subgroup of the tree, which displays that PpACS1 has the closest 
evolutional relationship with PcACS2b (El-Sharkawy et al., 2004), and relatively close relationship with PcACS2a (El-Sharkawy et al., 2004). Whereas the four ACSs, including AtACS8 (Mayer et al., 1999), PbACS1B, pPPACS1 (Itai et al., 1999) and PcACS1b (El-Sharkawy et al., 2004), occupy another branch of the tree, which suggests that PpACS1 might diverge earlier from these ACSs during evolution.

\subsection{Isolation and Characterization of PpACS1 Gene}

One PpACS gene was amplified from the pear (P. pyrifolia) genome by PCR, using gene-specific primers. Sequence comparison between the cDNA and its corresponding gene revealed that PpACS1 gene contains two introns splitting their ORF into three exons. The first intron is located within codons encoding valine, and the second intron is inserted within codons encoding phenylalanine in the gene (Fig.4).

\subsection{PpACS1 Gene is Preferentially Expressed in Leaves}

To analyse the expression patterns of the isolated PpACS1 gene, mRNA levels of the gene in pear tissues were quantified by RT-PCR using gene-specific primers. As shown in Fig.5, PpACS1 showed leaf-preferential expression pattern. PpACS1 was preferentially expressed in leaves, and moderate expression was found in anthers, but relatively weak signals were detected in shoots of pear. Little expression signal was detected in petals and developing fruit in pear.

\section{Discussion}

Pear and apple $A C S$ genes are highly homologous throughout the protein coding regions but do show a degree of sequence divergence within the 3' untranslated regions. The data presented in Fig.1 revealed that pear ACS proteins contain the conserved structure, Aminotransferase class I and II domain and Aminotransferases class-I pyridoxal-phosphate attachment site, like all the other ACS proteins. The structure of the Plant 1-aminocyclopropane-1-carboxylate (ACC) synthase genes has been studied. For example, the pear ACC synthase gene has three exons and two introns, with all of the boundaries between these introns and exons sharing a consensus dinucleotide sequence of GT-AG.

Previous studies revealed that $A C S$ genes display their tissue-specific or preferential expression in plants. PnACS from Pharbitis nil was expressed in cotyledons, petioles, hypocotyls, root and shoot apexes both in light- and dark-grown seedlings. The highest expression level of PnACS was found in the roots. The expression of PnACS is up-regulated in all the organs examined under IAA applied to the cotyledons of $P$. nil seedlings. In this case, the most IAA-responsive were the hypocotyls (Frankowski et al., 2009). Likewise, our results also revealed that PpACS1 gene was predominantly expressed in leaves, moderate expression was found in anthers and shoots, but little signals were detected in developing fruit (Fig.5), suggesting that it may be involved in early growth and development of pear.

\section{References}

Botella, J. R., Cavallaro, A. S., \& Cazzonelli, C. I. (2000). Towards the production of transgenic pineapple to control flowering and ripening. Acta Horticulturae, 529, 115-122.

Cazzonelli, C. I., Cavallaro, A. S., \& Botella, J. R. (1998). Cloning and characterisation of ripening-induced ethylene biosynthetic genes from non-climacteric pineapple (Ananas comosus) fruits. Australian Journal of Plant Physiology, 25, 513-518. http://dx.doi.org/10.1071/PP98013

El-Sharkawy, I., Jones, B., Gentzbittel, L., Lelie'vre, J. M., Pech, C., \& Latche, A. (2004). Differential regulation of ACC synthase gene in cold-dependent and -independent ripening in pear fruit. Plant Cell and Environment, 27, 1197-1210. http://dx.doi.org/10.1111/j.1365-3040.2004.01218.x

El-Sharkawy, I., Kim, W.S., Jayasankar, S., Svircev, A.M., \& Brown, D. C. W. (2008). Differential regulation of four members of the ACC synthase gene family in plum. Journal of Experimental Botany, 59(8), 2009-2027. http://dx.doi.org/10.1093/jxb/ern056

Frankowski, K., Kesy, J., Wojciechowski, W., \& Kopcewicz, J. (2009). Light- and IAA-regulated ACC synthase gene (PnACS) from Pharbitis nil. and its possible role in IAA-mediated flower inhibition. Journal of Plant Physiology, 166(2), 192-202. http://dx.doi.org/10.1016/j.jplph.2008.02.013

Itai, A., Kawata, T., Tanabe, K., Tamura, F., Uchiyama, M., Tomomitsu, M., \& Shiraiwam N. (1999). Identification of 1-aminocyclopropane-1-carboxylic acid synthase genes controlling the ethylene level of ripening fruit in Japanese pear (Pyrus pyrifolia Nakai). Molecular and General Genetics, 261(1), 42-49. http://dx.doi.org/10.1007/s004380050939

Klee, H., \& Tieman, D. (2002). The tomato ethylene receptor gene family: form and function. Physiologia 
Plantarum, 115, 336-341. http://dx.doi.org/10.1034/j.1399-3054.2002.1150302.x

Lambert, C., Leonard, N., De Bolle, X., \& Depiereux, E. (2002). ESyPred3D: prediction of proteins 3D structures. Bioinformatics, 18, 1250-1256. http://dx.doi.org/10.1093/bioinformatics/18.9.1250

Mathooko, F. M., Mwaniki, M. W., Nakatsuka, A., Shiomi, S., Kubo, Y., Inaba, A., \& Nakamura, R. (1999). Expression characteristics of CS-ACS1, CS-ACS2 and CS-ACS3, three members of the 1-aminocycIopropane-1-carboxylate synthase gene family in cucumber (Cucumis sativus L.) fruit under carbon dioxide stress. Plant Cell Physiology, 40(2), 164-172.

Mathooko, F. M., Tsunashima, Y., Owino, W. Z. O., Kubo, Y., \& Inaba, A. (2001). Regulation of genes encoding ethylene biosynthetic enzymes in peach (Prunus persica L.) fruit by carbon dioxide and 1-methylcyclopropene. Postharvest Biology and Technology, 21, 265-281. http://dx.doi.org/10.1016/S0925-5214(00)00158-7

Mayer, K., Schuller, C., Wambutt, R., Murphy, G., \& Volckaert, G. (1999). Sequence and analysis of chromosome 4 of the plant Arabidopsis thaliana. Nature, 402 (6763), 769-777. http://dx.doi.org/10.1038/47134

Nakatsuka, A., Shiomi, S., Kubo, Y., \& Inaba, A. (1997). Expression and internal feedback regulation of ACC synthase and ACC oxidase genes in ripening tomato fruit. Plant Cell Physiology, 38, 1103-1110.

Rodrigues-Pousada, R. A., Rycke, R .D., Dedonder, A., Van Caeneghem, W., Engler, G., Van Montagu, M., \& Van Der Straetena, D. (1993). The Arabidopsis 1-aminocyclopropane-1-carboxylate synthase gene 1 is expreked during early development. The Plant Cell, 5, 897-911. http://dx.doi.org/10.1105/tpc.5.8.897

Rosenfield, C. L., Kiss, E., \& Hrazdina, G. (1996). MdACS-2 (accession no. U73815) and Md-ACS3 (accession no. U73816): two new 1-aminocyclopropane-1-carboxylate synthases in ripening apple fruit. Plant Physiology, $112,1735$.

Salman-Minkov, A., Levi, A., Wolf, S., \& Trebitsh, T. (2008). ACC synthase genes are polymorphic in watermelon (Citrullus spp.) and differentially expressed in flowers and in response to auxin and gibberellin. Plant Cell Physiology, 49(5), 740-750. http://dx.doi.org/10.1093/pcp/pen045

Shi Haiyan, Zhu Li, Zhou Ying, Li Gang, Chen Liang, \& Li Xuebao. (2009). A cotton gene encoding a polygalacturonase inhibitor-like protein is specifically expressed in petals. Acta Biochimica et Biophysica Sinica, 41(4), 316-324. http://dx.doi.org/10.1093/abbs/gmp020

Tatsuki, M., Haji, T., \& Yamaguchi, M. (2006). The involvement of 1-aminocyclopropane-1-carboxylic acid synthase isogene, Pp-ACS1, in peach fruit softening. Journal of Experimental Botany, 57(6), 1281-1289. http://dx.doi.org/10.1093/jxb/erj097

Trusov, Y., \& Botella, J.R. (2006). Silencing of the ACC synthase gene ACACS2 causes delayed flowering in pineapple [Ananas comosus (L.) Merr.]. Journal of Experimental Botany, 57(14), 3953-3960. http://dx.doi.org/10.1093/jxb/erl167

Ververidis, P., \& John, P. (1991). Complete recovery in vitro of ethylene forming enzymes activity Phytochemistry, 30, 725-727. http://dx.doi.org/10.1016/0031-9422(91)85241-Q

Wang, A. D., Yamakake, J., Kudo, H., Wakasa, Y., Hatsuyama, Y., Igarashi, M., Kasai, A., Li, T. Z., \& Harada, T. (2009). Null mutation of the MdACS3 gene, coding for a ripening-specific 1-aminocyclopropane-1-carboxylate synthase, leads to long shelf life in apple fruit. Plant Physiology, 151 (1), 391-399. http://dx.doi.org/10.1104/pp.109.135822

Wang, N. N., Shih, M. C., \& Li, N. (2005). The GUS reporter-aided analysis of the promoter activities of Arabidopsis ACC synthase genes AtACS4, AtACS5, and AtACS7 induced by hormones and stresses. Journal of Experimental Botany, 56(413), 909-920. http://dx.doi.org/10.1093/jxb/eri083

Yang, S. F., \& Hoffman, N. E. (1984). Ethylene biosynthesis and its regulation in higher plants. Annual Review of Plant Physiology, 35, 155-189. http://dx.doi.org/10.1146/annurev.arplant.35.1.155 


\begin{tabular}{|c|c|c|}
\hline PpACS1 & MAIDIEQQQQPSPGLSKIAVSDTHGEDSPYFAGWKTYDENPYHESSNPSGVIQMGLAENQ & 60 \\
\hline pPPACS2 & MAIDIEQQQQPSPGLSKIAVSDTHGEDSPYFAGWKAYDENPYHESSNPSGVIQMGLAENQ & 60 \\
\hline PcACS3a & MAIDIEQQQQPSPGLSKIAISDTHGEDSPYFAGWKAYDENPYHESSNPSGVIQMGLAENQ & 60 \\
\hline PcACS2b & MAIDIEQRKQPSPRLSKIAVSDTHGEDSPYFAGWKTYDENPYHESSNPSGVIQMGLAENQ & 60 \\
\hline $\operatorname{MdACS} 3 \mathrm{a}-1$ & MAIDIEQRQQPSPGLSKIAVSDTHGEDSPYFAGWKAYDENPYHESSNPSGVIQMGLAENQ & 60 \\
\hline $\operatorname{MdACS} 3 \mathrm{a}-2$ & MAIDIEQRQQPSPGLSKIAVSDTHGEDSPYFAGWKAYDENPYHESSNPSGVIQMGLAENQ & 60 \\
\hline PpACS1 & VSFDLLENHLEENCEASTWGSKGSKGVSGFRENALFQDYHGLLSFRKAMASFMEQIRGGR & 120 \\
\hline pPPACS2 & VSFDLLEKHLEENSEASNWGSKGSKGVSGFRENALFQDYHGLLSFRKAMASFMEQIRGGR & 120 \\
\hline PcACS3a & VSFDLLEKHLEENSEASNWGSKGSKGASSGFRENALFQDYHGLLSYKKAMANFMEQIRGGR & 120 \\
\hline PcACS2b & VSFDLLENHLEENSEASTWGSKGSKGVSGFRENALFQDYHGLLSFRKAMASFMEQIRGGR & 120 \\
\hline $\operatorname{MdACS} 3 \mathrm{a}-1$ & VSFDLLEKHLEENSEASNWGSKGTKGVSGFRENALFQDYHGLLSFRKAMANFMEQIRGGR & 120 \\
\hline $\operatorname{MdACS} 3 \mathrm{a}-2$ & VSFDLLEKHLEENSEASNWGSKGSKGVSGFRENALFQDYHGLLSFRKAMANFMEQIRGGR & 120 \\
\hline PpACS1 & AKFDPARVVLTAGATAANELLTFVIADPGDALLVPTPYYPGFDRDLRWRTGVNIVPIHCE & 180 \\
\hline pPPACS2 & $\begin{array}{l}\text { AKFDPARVVLTAGATAANELLTFI IADPGDALLVPTPYYPGFDRDLRWRTGVNIVPIHCE } \\
\end{array}$ & 180 \\
\hline PcACS3a & AKFDPVRVVLTAGATAADELLTFI IADPGDALLVPTPYYPGFDRDLRWRTGVNIVPIHCE & 180 \\
\hline PcACS2b & AKFDPARVVLTAGATAANELLTFVIADPGDALLVPTPYYPGFDRDLRWRTGVNIVPIHCE & 180 \\
\hline $\operatorname{MdACS} 3 \mathrm{a}-1$ & AKFDPVRVVLTAGATAANELLTFIIADPGDALLVPTPYYPGFDRDLRWRTGVNIVPIHCE & 180 \\
\hline $\operatorname{MdACS} 3 \mathrm{a}-2$ & AKFDPVRVVLTAGATAANELLTFI IADPGDALLVPTPYYPGFDRDLRWRTGVNIVPIHCE & 180 \\
\hline PpACS1 & SSNNFQITPQALEAAYKEAEAKNMRVRGVLFTNPSNPLGATIQRTVLEEILDFVTQKNIH & 240 \\
\hline pPPACS2 & SSNNFQITPQALEAAYKEAEAKNMRVRGVLITNPSNPLGATIQRAVLEEILDFVTQKNIH & 240 \\
\hline PcACS3a & SSNNFQITPQALEAAYKEAEARNMRVRGVLFTNPSNPLGATIQRTVLEEILDFVTQKNIH & 240 \\
\hline PcACS2b & SSNNFQITPQALEAAYKEAEAKNMRVRGVLFTNPSNPLGATIQRTVLEEILDFVTQKNIH & 240 \\
\hline $\operatorname{MdACS} 3 \mathrm{a}-1$ & $\begin{array}{l}\text { SSNNFQITPQALEAAYKEAEAKNMRVRGVLITNPSNPLGATIQRAVLEEILDFVTQKNIH } \\
\end{array}$ & 240 \\
\hline MdACS3a-2 & SSNNFQITPQALEAAYKEAEAKNMRVRGVLITNPSNPLGATIQRAVLEEILDFVTQKNIH & 240 \\
\hline PpACS1 & LVSDEIYSGSAFSSSEFISVAEILEDRQYKDAERVHIVYSLSKDLGLPGFRVGTVYSYND & 300 \\
\hline pPPACS2 & LVSDEIYSGSAFSSSEFISVAEILEDRQYKDAERVHIVYSLSKDLGLPGFRVGTVYSFEND & 300 \\
\hline PcACS3a & LVSDEVYSGSAFSSSEFISVAEIMEDRQYKDAERVHIVYSLSKDLGLPGFRVGTVYSYND & 300 \\
\hline PcACS2b & LVSDEIYSGSAFSSSEFISVAEILENRQYKDAERVHIVYSLSKDLGLPGFRVGTVYSYND & 300 \\
\hline $\operatorname{MdACS} 3 \mathrm{a}-1$ & LVSDEIYSGSAFSSSEFISVAEILEDRQYKDAERVHIVYSLSKDLGLPGFRVGTVYSYND & 300 \\
\hline MdACS3a-2 & LVSDEIYSGSAFSSSEFISVAEILEDRQYKDAERVHIVYSLSKDLGLPGFRVGTVYSYND & 300 \\
\hline PpACS1 & KVVTTARRMSSFTLISSQTQHLLASMLSDKEFTGNYINTNRERLRRRYDMIVEGLKKSGI & 360 \\
\hline pPPACS2 & KVVTTARRMSSFTLISSETQHLLASMLSDKEFTGNY IKTNRERLRRRYDMIVEGLKKSGI & 360 \\
\hline PcACS3a & KVVTTARRMSSFTLISSQTQHLLASMLSDKEFTENYIKTNRERLRRRYDMIVEGLKKSGI & 360 \\
\hline PcACS2b & KVVTTARRMSSFTLISSQTQHLLASMLSDKEFTENY IKTNRERLRRRYDMILEGLKKSGI & 360 \\
\hline $\operatorname{MdACS} 3 \mathrm{a}-1$ & KVVTTARRMSSFTLISSQTQHLLASMLSDKEFTGNY IKTNRERLRTRYDMIVQGLKKSGI & 360 \\
\hline MdACS3a-2 & KVVTTARRMSSFTLISSQTQHLLASMLSDKEFTGNYIKTNRERLRTRYDMIIEGLKKSGI & 360 \\
\hline PpACS1 & ECLKGNAGLFCWMNLSPFLDEPTRECELTLWDSMLHEVKLNISPGSSCHCSEPGWFRVCF & 420 \\
\hline pPPACS2 & ECLKGNAGLFCWMNLSPFLDEPTRECELTLWDSMLHEVKLNISPGSSCHCSEPGWFRVCF & 420 \\
\hline PcACS3a & ECLKGNAGLFCWMNLSPFLDEPTRESELTLWDSMLHEVKLNISPGSSCHCSEPGWFRVCF & 420 \\
\hline PcACS2b & ECLKGNAGLFCWMNLSPFLDEPTRESELTLWDSMLHEVKLNISPGSSCHCSEPGWFRVCF & 420 \\
\hline $\operatorname{MdACS} 3 \mathrm{a}-1$ & ECLKGNAGLFCWMNLSPFLDEPTRECELTLWDSMLHEVKLNISPGSSCHCSEPGWFRVCF & 420 \\
\hline MdACS3a-2 & ECLKGNAGLFCWMNLSPFLDEPTRECELTLWDSMLHEVKLNISPGSSCHCSEPGWFRVCF & 420 \\
\hline PpACS1 & ANMSEQTLGVALTRIHNFMEKRERAC 446 & \\
\hline pPPACS2 & ANMSEQTLGIALTRIHNFMEKRERAC 446 & \\
\hline PcACS3a & ANMSEQTLGIALTRIHNFMEKRERAC 446 & \\
\hline PcACS2b & ANMSEQTLGIALTRIKNFMEKRERAC 446 & \\
\hline $\operatorname{MdACS} 3 \mathrm{a}-1$ & ANMSEQTLGIALTRIHNFMEKRERAC 446 & \\
\hline MdACS3a-2 & ANMSEQTLGIALTRIHNFMEKRERAC 446 & \\
\hline
\end{tabular}

Figure 1. Sequence alignment among the pear and apple ACS proteins 
Sequences of pear and apple ACSs were aligned. Amino acid substitutions are highlighted in black and in grey. Arrows indicate the positions of the two introns in pear PpACS1 genes. Aminotransferase class I and II domains are underlined. Aminotransferases class-I pyridoxal-phosphate attachment sites are boxed. N-glycosylation site is bold; cAMP- and cGMP-dependent protein kinase phosphorylation site is italic. The accession numbers of pear and apple ACS proteins in GenBank are: PpACS1 (JQ284383), pPPACS2 (BAA76388), PcACS3a (AAR12136), PcACS2b (AAR38503), MdACS3a-1 (AEP82200), and MdACS3a-2 (AEP82201). Pp: Pyrus pyrifolia; Pc: Pyrus communis; Md: Malus domestica

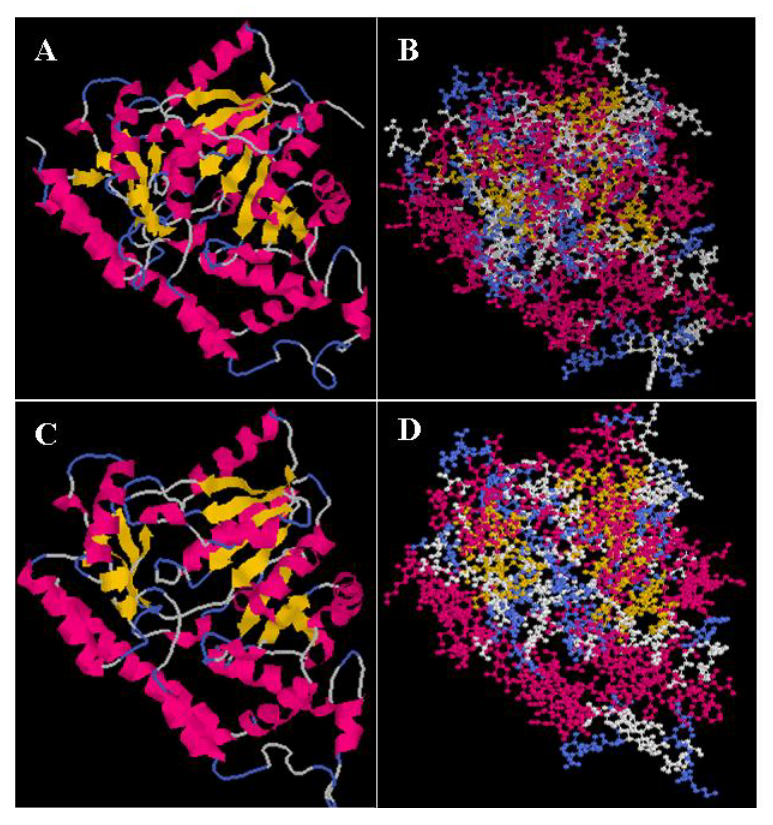

Figure 2. Three dimensional structure of the predicted PpACS1 protein from side views

(A, B) Illustration of the full PpACS1 models from cartoon and ball and stick views. (C, D) Aminotransferase class I and II domain models from cartoon and ball and stick views. 


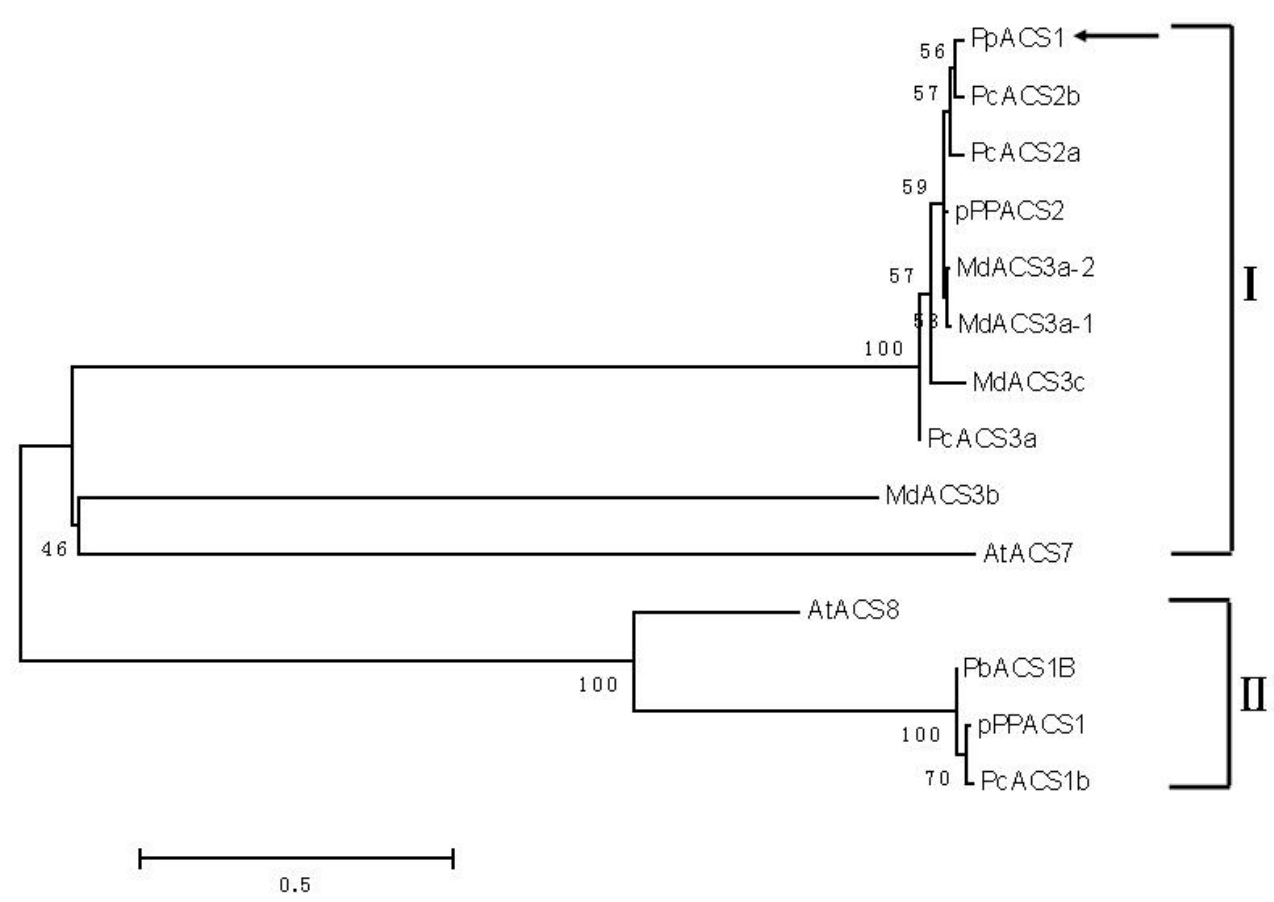

Figure 3. Phylogenetic relationship of pear ACS proteins to apple and Arabidopsis ACS proteins

The minimum evolution tree was constructed in MEGA3.1 from 1000 bootstrap replicates. The accession numbers of pear, apple and Arabidopsis ACS proteins in GenBank are: PpACS1 (Pyrus pyrifolia, JQ284383), PcACS2b (Pyrus communis, AAR38503), PcACS2a (Pyrus communis, AAL66201), pPPACS2 (Pyrus pyrifolia, BAA76388), MdACS3a-2 (Malus $x$ domestica, AEP82201), MdACS3a-1 (Malus $x$ domestica, AEP82200), MdACS3c (Malus $x$ domestica, BAE94692), PcACS3a (Pyrus communis, AAR12136), MdACS3b (Malus $x$ domestica, BAE94691), AtACS7 (Arabidopsis thaliana, NP_194350), AtACS8 (Arabidopsis thaliana, NP_195491), PbACS1B (Pyrus x bretschneideri, BAF44094), pPPACS1 (Pyrus pyrifolia, BAA76389) and PcĀCS1b (Pyrus communis, AAR38501). Pp: Pyrus pyrifolia; Pb: Pyrus x bretschneideri; Pc: Pyrus communis; Sl: Solanum lycopersicum

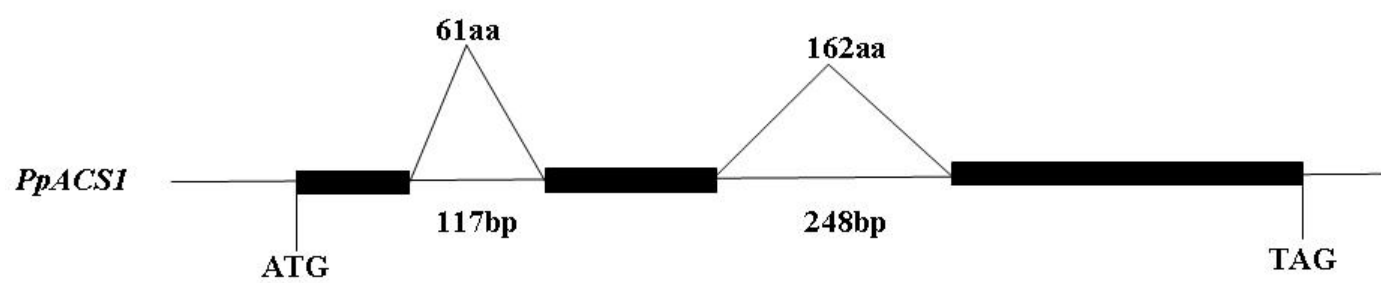

Figure 4. The structure of PpACS1 gene

Exons are denoted by black boxes. Introns, $5^{\prime}$-flanking regions, and $3^{\prime}$-UTRs are denoted by lines. The length of the intron in base pairs is indicated. The position of substitution is denoted by a diagonal line.

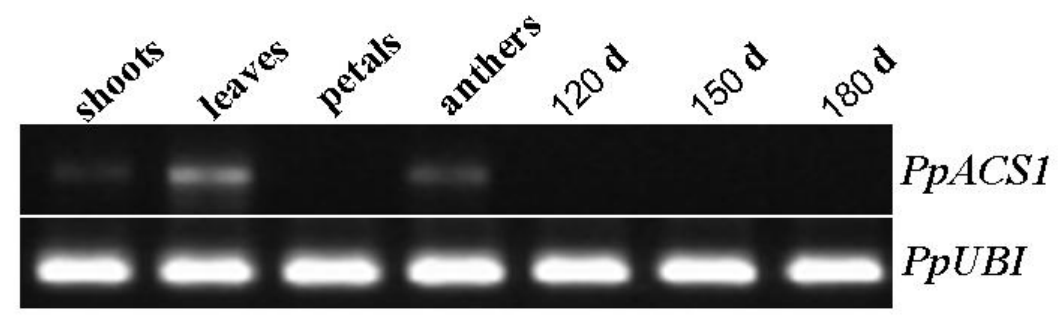

Figure 5. RT-PCR analysis of expression of PpACS1 gene in pear tissues

$P p U B I$ was amplified as a control. $120 \mathrm{~d}, 150 \mathrm{~d}$, and $180 \mathrm{~d}$ refer to 120,150 , and 180 day-old after full bloom fruit. 